\title{
THE PROPAGATION OF QUANTUM RELATIVISTIC WAVEPACKETS IN ELECTROMAGNETIC FIELDS*
}

\author{
P. R. Johnson ${ }^{\dagger}$, A. J. Dragt ${ }^{\ddagger}$ \\ University of Maryland, College Park, MD 20742, USA
}

\begin{abstract}
We describe the simulation of a quantum relativistic wavepacket propagating in electric and magnetic fields. We start from first principles in QED with the intent of exploring the effects of spin, extended wavepackets, and radiation reaction on particle motion. (This initial work neglects radiation reaction and anti-particle effects). In the numerical simulation of wavepackets we use unitary integrators, adapted to particle propagation in a background (though possibly time-dependent) vector potential.
\end{abstract}

\section{INTRODUCTION}

The use of symplectic integrators in the computation of charged particle classical orbits allows the numerical simulation of trajectories preserving the canonical structure of classical mechanics; high-order symplectic integrators may be systematically produced by methods such as those introduced by Ruth, Neri, Forest, Yoshida, Suzuki, and others [1]. But the classical (Hamiltonian) dynamics of particle motion is of course only an approximation (presumably) describing the time-dependent expectation values of appropriate operators with respect to the quantum state of the relativistic particle.

New issues for particle motion, of both a fundamental and practical nature, arise within the framework of quantum theory. For instance, dissipation from radiation reaction, which is not generated by a Hamiltonian vector field, is a significant process in the context of synchrotron radiation. While classical dissipative processes may be generated from non-Hamiltonian vector fields [2], the classical theory of radiation reaction for point particles is inconsistent (e.g. it gives acausal and runaway solutions), and so the correct model of classical radiation reaction is an open question. One should therefore use the quantum theory of radiation reaction to determine both the correct classical limit and quantum corrections to that limit.

Of course, even before considering radiation reaction (which does not appear until third order in QED perturbation theory) there are uniquely quantum phenomena arising at lower orders that may give significant corrections to the long-time dynamics of charged particles moving in background electric and magnetic fields. This includes both the influence of spin, and the fact that evolving quantum wavepackets are extended in space and thus depend on the

\footnotetext{
${ }^{*}$ Work supported in part by DOE Grant DEFG0296ER40949

† philipj@physics.umd.edu

$\ddagger$ dragt@physics.umd.edu
}

values of the background fields in the entire region that the wavepacket occupies.

In this paper, we describe our method for simulating the quantum dynamics of charges moving in background fields neglecting, for the present, higher order effects like radiation reaction. We make use of unitary integrators to ensure consistent quantum dynamics of the particle state. The next stage following this preliminary work is to move these simulations onto parallel computing architectures where we can study in detail particle quantum dynamics in typical magnetic fields.

\section{RELATIVISTIC WAVEPACKETS}

We shall consider one-particle states that are a superposition of momentum states $|\mathbf{p}, s\rangle=\left(2 E_{\mathbf{p}}\right)^{1 / 2} \hat{a}_{s}^{\dagger}(\mathbf{p})|v a c\rangle$, where $s= \pm$ is the spin polarization in the $\pm \hat{z}$ direction, and $\hat{a}_{s}^{\dagger}(\mathbf{p})$ is the one-particle creation operator. (We set $\hbar=c=1$, so $E_{\mathbf{p}}$ is the energy). Standard relativistic quantum field theory, unlike nonrelativistic quantum mechanics, does not have a position operator $\hat{x}(t)$. Instead, computing the motion of a quantum relativistic particle requires first evolving the particle state, and then computing the expectation values of appropriate field-observables. For example, the charged-density expectation value $\left\langle f(t)\left|\hat{j}^{0}(\mathbf{x})\right| f(t)\right\rangle$ is proportional to the probability density for a single particle, where $\hat{\jmath}^{\mu}(\mathbf{x})=\bar{\psi}(\mathbf{x}) \gamma^{\mu} \hat{\psi}(\mathbf{x})$ is the current density for the Dirac field $\hat{\psi}(\mathbf{x})$ in the Schrödinger picture, $\gamma^{\mu}$ are the Dirac matrices (in the Dirac-Pauli representation), and the adjoint is defined by $\bar{\psi}(\mathbf{x}) \equiv \hat{\psi}^{\dagger}(\mathbf{x}) \gamma^{0}$.

Strained crystals can produce spin-polarized electrons; we will assume that a typical electron is ejected with its spin polarized in the $+\hat{z}$-direction and with its wavepacket state given as

$$
\left|f_{0}\right\rangle=\int d \mu(\mathbf{p}) f_{0}(\mathbf{p})|\mathbf{p},+\rangle .
$$

Generalizing to unpolarized states is straightforward. $f_{0}(\mathbf{p})$ is a normalized Gaussian centered around $\mathbf{p}_{0}$ of momentum space width $\lambda_{p}$. For brevity we define $d \mu(\mathbf{p})=$ $(2 \pi)^{-3}\left(2 E_{\mathbf{p}}\right)^{-1 / 2} d^{3} p$. We then boost by $\Lambda=\Lambda(\overline{\mathbf{p}})$ (with $\left.|\overline{\mathbf{p}}| \gg\left|\mathbf{p}_{0}\right|\right)$ in the $+\hat{z}$-direction producing the relativistic electron state

$$
|f\rangle=\hat{U}(\Lambda)\left|f_{0}\right\rangle=\int d \mu(\mathbf{p}) f(\mathbf{p})|\mathbf{p},+\rangle,
$$

where $f(\mathbf{p})=f_{0}\left(\Lambda^{-1} \mathbf{p}\right)$ is the boosted packet centered around momentum $\overline{\mathbf{p}} . \hat{U}(\Lambda)$ is the unitary operator generating the boost $\Lambda$ in Hilbert space, and we have used the 
Lorentz invariance of the measure: $d \mu\left(\Lambda^{-1} \mathbf{p}\right)=d \mu(\mathbf{p})$. Note that $|f\rangle$ is nearly a helicity eigenstate up to corrections of order $\left|\mathbf{p}_{0}\right| /|\overline{\mathbf{p}}| \ll 1$.

\section{COMPUTING POSITION, CURRENT, AND SPIN EXPECTATION VALUES}

So long as the single particle theory is adequate, in other words, so long as pair creation is negligible, we may drop the anti-particle part of the field $\hat{\psi}(\mathbf{x})$, and write

$$
\hat{\psi}(\mathbf{x}) \rightarrow \hat{\psi}^{(+)}(\mathbf{x})=\sum_{r} \int d \mu(\mathbf{p}) e^{i \mathbf{p} \cdot \mathbf{x}} u_{r}(\mathbf{p}) \hat{a}_{r}(\mathbf{p}) .
$$

$u_{r}(\mathbf{p})$ are positive energy Dirac spinors with normalization $u_{r}^{\dagger}(\mathbf{p}) u_{r^{\prime}}(\mathbf{p})=2 E_{\mathbf{p}} \delta_{r r^{\prime}}$. In the Dirac-Pauli representation of the gamma-matrix algebra the spinors $u_{ \pm}$are

$$
u_{ \pm}(\mathbf{p})=\left(\gamma^{\mu} p_{\mu}+m\right) \chi_{ \pm}
$$

where $\chi_{+}=(1,0,0,0)$ and $\chi_{-}=(0,1,0,0)$ give $\pm \hat{z}$-spin for a particle at rest.

The spinor-valued configuration space function wavepacket is given by

$$
\begin{aligned}
f_{a}(\mathbf{x}, t) & \equiv\left\langle v a c\left|\hat{\psi}_{a}^{(+)}(\mathbf{x})\right| f(t)\right\rangle \\
& =\int d \mu(\mathbf{p}) e^{i \mathbf{p} \cdot \mathbf{x}} f(\mathbf{p}, t) u_{+}(\mathbf{p}) \\
& =(2 \pi)^{-3} \int d \mathbf{p} e^{i \mathbf{p} \cdot \mathbf{x}} \varphi_{a}(\mathbf{p}, t)
\end{aligned}
$$

where $a=(1, \ldots, 4)$ is a spinor index, and $|f(t)\rangle=$ $\hat{U}(t)|f\rangle$ is the time-evolving state in the Schrödinger picture $(\hat{U}(t)$ is the unitary time-evolution operator). We have defined

$$
\varphi_{a}(\mathbf{p}, t)=\left(2 E_{\mathbf{p}}\right)^{-1 / 2} f(\mathbf{p}, t) u_{+}(\mathbf{p}) .
$$

From the normalization of $u_{r}(\mathbf{p})$ it follows that $\varphi^{\dagger}(\mathbf{p}, t) \varphi(\mathbf{p}, t)=|f(\mathbf{p}, t)|^{2}$.

In terms of $f(\mathbf{x}, t)$, the four-current expectation value is

$$
\left\langle f(t)\left|\hat{\jmath}^{\mu}(\mathbf{x})\right| f(t)\right\rangle=\bar{f}(\mathbf{x}, t) \gamma^{\mu} f(\mathbf{x}, t) .
$$

Using $\gamma^{0} \gamma^{0}=1$ the charge density is

$$
\left\langle f(t)\left|\hat{\jmath}^{0}(\mathbf{x})\right| f(t)\right\rangle=f^{\dagger}(\mathbf{x}, t) f(\mathbf{x}, t),
$$

and other expectation values may be similarly computed. The initial wavepacket $\left|f_{0}(\mathbf{p})\right|^{2}$ (with $\mathbf{p}_{0}=0$ ) is plotted in Fig. 1a. The charge-probability density $\left\langle f\left|\hat{\jmath}^{0}(\mathbf{x})\right| f\right\rangle$ for the wavepacket after a boost of $\beta=v / c=.95$ in the $\hat{z}$-direction is plotted in Fig. 1b. The figures show the wavepackets intersection with the $\mathbf{p}_{\mathbf{x}}=0$ and $\mathbf{x}=0$ planes, respectively. Notice the Lorentz contraction generated flattening in the $\hat{z}$-direction of the boosted wavepacket .

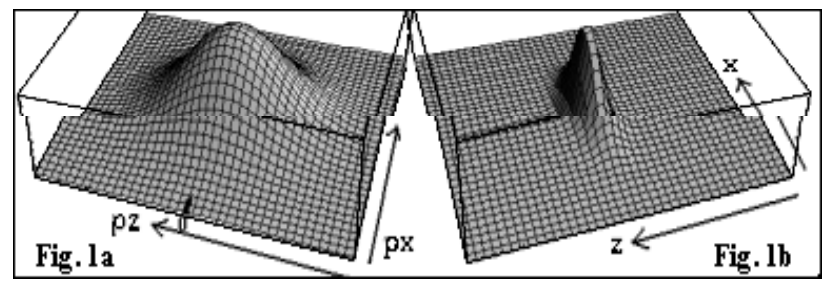

Figure 1: Fig. 1a shows $\left|f_{0}(\mathbf{p})\right|^{2}$ for an initial Gaussian wavepacket of average momentum $\mathbf{p}_{0}=0$. Fig. $1 \mathrm{~b}$ shows the charge-probability density $\left\langle f\left|\hat{\jmath}^{0}(\mathbf{x})\right| f\right\rangle$ for the wavepacket after a boost of $\beta=.95$ in the $\hat{z}$-direction.

\section{EQUATIONS OF MOTION FOR SINGLE PARTICLES IN TIME-DEPENDENT FIELDS}

The (functional) Schrödinger equation is

$$
i \partial_{t}|f(t)\rangle=\hat{H}(t)|f(t)\rangle
$$

where

$$
\begin{aligned}
\hat{H}(t) & =\int d \mathbf{x} \hat{\psi}^{\dagger}(\mathbf{x}) H_{D}(\mathbf{x}, t) \hat{\psi}(\mathbf{x}) \\
& =\int d \mathbf{x} \hat{\psi}^{\dagger}(\mathbf{x})(A(\mathbf{p})+B(\mathbf{x}, t)) \hat{\psi}(\mathbf{x}) \\
& \equiv \hat{A}+\hat{B}(t)
\end{aligned}
$$

is the field Hamiltonian, and $H_{D}(\mathbf{x}, t)=A(\mathbf{p})+$ $B(\mathbf{x}, t)$, with $A(\mathbf{p})=\gamma^{0}\left(\gamma^{i} \mathbf{p}_{i}+m\right)$ and $B(\mathbf{x}, t)=$ $e \gamma^{0} \gamma^{\mu} A_{\mu}(\mathbf{x}, t)$, is the matrix-valued Dirac Hamiltonian $(\mathbf{p}=-i \nabla)$. We use hats, e.g. $\hat{H}$, to distinguish fieldoperators from quantum mechanical operators, e.g. $H_{D}$.

When it is valid to restrict to the one particle sector, we may show by operating from the left with $\langle v a c| \hat{\psi}_{a}^{(+)}(\mathbf{x})$ on both sides of Eq. 2 that $f(\mathbf{x}, t)$ satisfies the Dirac equation:

$$
i \frac{\partial}{\partial t} f(\mathbf{x}, t)=H_{D}(\mathbf{x}, t) f(\mathbf{x}, t)
$$

whose formal solution is

$$
\begin{aligned}
f(\mathbf{x}, t) & =U(t) f(\mathbf{x}) \\
U(t) & =T e^{-i \int{ }^{t} H_{D}\left(\mathbf{x}, t^{\prime}\right) d t^{\prime}} .
\end{aligned}
$$

$T$ denotes time-ordering. For simplicity, we set $t_{0}=0$, and write $f(\mathbf{x})=f\left(\mathbf{x}, t_{0}\right)$ and $U(t)=U\left(t, t_{0}\right)$.

\section{TIME-INDEPENDENT EVOLUTION IN EXTENDED HILBERT SPACE}

We now apply to Hilbert space the analog of extending phase space with $t$ and $p_{t}$ as canonically conjugate dynamical variables. This allows us to make use of higher-order unitary integration schemes developed for time-independent Hamiltonians. Promoting $t$ to an operator $\hat{t}$, we introduce its canonical momentum $\hat{p}_{t}$ such that 
$\left[\hat{t}, \hat{p}_{t}\right]=i \hbar$ and $[\hat{t}, \hat{\psi}(\mathbf{x})]=0$. The operator $\hat{t}$ has eigenstates $|t\rangle$ such that $\hat{t}|t\rangle=t|t\rangle$, and the extended Hilbert space $\mathcal{K}$ is spanned by $|f, t\rangle=|f\rangle \otimes|t\rangle$. Since $\hat{p}_{t}$ is conjugate to $\hat{t}$, it generates time translations via

$$
e^{-i \hat{p}_{t} \varepsilon}|t\rangle=|t+\varepsilon\rangle \text {. }
$$

If $B$ is a time-dependent function of field operators $\hat{\psi}$, in the extended quantum theory we have $B(\hat{\psi}, t) \rightarrow B(\hat{\psi}, \hat{t})$ and therefore

$$
B(\hat{\psi}, \hat{t})\left|f, t^{\prime}\right\rangle=B\left(\hat{\psi}, t^{\prime}\right)\left|f, t^{\prime}\right\rangle .
$$

Evolution with respect to the new parameter $\tau$ in the Hilbert space $\mathcal{K}$ is generated by the $\tau$-independent Hamiltonian $\hat{K}=\hat{p}_{t}+\hat{H}$. The extended (functional) Schrödinger equation becomes

$$
i \partial_{\tau}|f, t\rangle=\hat{K}|f, t\rangle
$$

with solution

$$
|f, t ; \tau\rangle=e^{-i \hat{K} \tau}|f, t\rangle .
$$

Now it follows from $d \hat{t} / d \tau=-i \hbar^{-1}[\hat{t}, \hat{K}]=1$ that $\hat{t}|f, t\rangle=\tau|f, t\rangle$. Hence, the label $\tau$ in Eq. 3 is unnecessary and we may write $|f, t\rangle=e^{-i \hat{K} t}|f\rangle$.

\section{UNITARY INTEGRATORS}

Defining $\varepsilon=t / N$ ( $N$ an integer), we may use leap-frog factorization to write

$$
\begin{aligned}
e^{-i \hat{K} \varepsilon} & =e^{-i \varepsilon \hat{p}_{t} / 2} e^{-i \varepsilon \hat{A} / 2} e^{-i \varepsilon \hat{B}(\hat{t})} e^{-i \varepsilon \hat{A} / 2} e^{-i \varepsilon \hat{p}_{t} / 2} \\
& =e^{-i \varepsilon \hat{p}_{t}} e^{-i \varepsilon \hat{A} / 2} e^{-i \varepsilon \hat{B}(\hat{t}+\varepsilon / 2)} e^{-i \varepsilon \hat{A} / 2}
\end{aligned}
$$

plus $\mathcal{O}\left(\varepsilon^{3}\right)$ corrections. In writing Eq. 4 we use $e^{i \varepsilon \hat{p}_{t} / 2} g(\hat{\psi}, \hat{t}) e^{-i \varepsilon \hat{p}_{t} / 2}=g(\hat{\psi}, \hat{t}+\varepsilon / 2)$, and assume that $\hat{A}$ is independent of $\hat{t}$ so that $\left[\hat{p}_{t}, \hat{A}\right]=0$.

Concatenating Eq. $4 N$ times (using $t=N \varepsilon$ ), we find

$e^{-i \hat{K} t}=e^{-i t \hat{p}_{t}} e^{i \frac{\varepsilon}{2} \hat{A}}\left[\prod_{n=1}^{N} e^{-i \varepsilon \hat{A}} e^{-i \varepsilon \hat{B}\left(\hat{t}+\left(n-\frac{1}{2}\right) \varepsilon\right)}\right] e^{-i \frac{\varepsilon}{2} \hat{A}}$.

This expression is organized to minimize subsequent computations. Therefore, $2^{n d}$-order equations of motion for the one-particle spinor wavepacket are

$$
\begin{aligned}
f(\mathbf{x}, t) & \equiv\left\langle v a c\left|\hat{\psi}^{(+)}\right| f, t\right\rangle=\left\langle v a c\left|\hat{\psi}^{(+)} e^{-i \hat{K} t}\right| f\right\rangle \\
& =e^{i \frac{\varepsilon}{2} A}\left[\prod_{n=1}^{N} e^{-i \varepsilon A} e^{-i \varepsilon B\left(\left(n-\frac{1}{2}\right) \varepsilon\right)}\right] e^{-i \frac{\varepsilon}{2} A} f(\mathbf{x})
\end{aligned}
$$

Time-translation invariance of the vacuum gives $\langle v a c| e^{-i t \hat{p}_{t}}=\langle v a c|$, and $t_{0}=0$ implies $\hat{t}|f\rangle=t_{0}|f\rangle=0$. Leap-frog factorization therefore yields the 'mid-point' rule of evaluating the (possible) time-dependent matrix $B$ at $B(t+\varepsilon / 2)$ during each $\varepsilon$ time step between $t$ to $t+\varepsilon$.

We may now generalize to higher-orders by standard methods. For example, Yoshida's symmetric $5^{\text {th }}$-order factorization implies

$$
\begin{aligned}
e^{-i \hat{K} \varepsilon} & =e^{-i \varepsilon \hat{p}_{t}} e^{i \frac{\varepsilon_{1}}{2} \hat{A}}\left(e^{-i \varepsilon_{1} \hat{A}} e^{-i \varepsilon_{1} \hat{B}\left(\hat{t}+\varepsilon-\frac{\varepsilon_{1}}{2}\right)}\right) \\
& \times\left(e^{-i\left(\varepsilon_{0}+\varepsilon_{1}\right) \frac{\hat{A}}{2}} e^{-i \varepsilon_{0} \hat{B}\left(\hat{t}+\frac{\varepsilon}{2}\right)}\right) \\
& \times\left(e^{-i\left(\varepsilon_{0}+\varepsilon_{1}\right) \frac{\hat{A}}{2}} e^{-i \varepsilon_{1} \hat{B}\left(\hat{t}+\frac{\varepsilon_{1}}{2}\right)}\right) e^{-i \frac{\varepsilon_{1}}{2} \hat{A}}
\end{aligned}
$$

plus $\mathcal{O}\left(\varepsilon^{5}\right)$ errors for each $\varepsilon$ step, with $\varepsilon_{1}=$ $\varepsilon /\left(2-2^{1 / 3}\right)>\varepsilon$ and $\varepsilon_{0}=-2^{1 / 3} \varepsilon_{1}<0$. Because $t+\varepsilon_{1} / 2>t+\varepsilon / 2>t+\varepsilon-\varepsilon_{1} / 2$, we get the well-know prescription of evaluating $B$ at successive 'backward-intime' steps during each interval $[t, t+\varepsilon]$ [3]. The operator $e^{-i \varepsilon_{1} \hat{A}}$ moves the state forward by $\varepsilon_{1}$ into the next interval where this 'time-reversed' pattern is repeated. Concatenating this result $N$ times gives a $4^{\text {th }}$-order unitary integrator for the interval $[0, t]$. Arbitrary higher-(even)-order integrators may be constructed by following Suzuki's [4] method combined with the extended Hilbert space structure outlined above.

\section{CONCLUSIONS}

Use of the above unitary integrators reduces simulation of the single particle wavepacket evolution to a sequence of alternating fast Fourier transforms (FFTs) and matrix multiplications. Each FFT diagonalizes the matrixexponentials in either configuration or momentum space, giving separate $4 \times 4$ matrix multiplications of the discretized spinor wavepacket $f_{a}(\mathbf{x})$ at every lattice point. The matrix-exponentials may be efficiently computed using scaling and squaring methods [2].

Work is currently in progress testing these methods on small lattices. On large lattices with small time steps, this problem quickly becomes numerically challenging, but the general structure of these simulations should lend itself to parallel computation.

\section{REFERENCES}

[1] R.D. Ruth, IEEE Trans. Nucl. Sci. NS-30, p.2669 (1983); F. Neri, Lie Algebras and Canonical Integration, University of Maryland Physics Department technical report, (1987); H. Yoshida, Phys. Lett. A 150, p. 262 (1990); E. Forest and R.D. Ruth, Physica D 43, p. 105 (1990); M. Suzuki, Phys. Lett. A 146, p. 319 (1990).

[2] A.J. Dragt, Lie Methods for Nonlinear Dynamics with Applications to Accelerator Physics, University of Maryland Physics Department technical report, (2001).

[3] M. Suzuki, J. Math. Phys. 32, p. 400 (1991).

[4] M. Suzuki, Phys. Lett. A 165, p. 387 (1992).

[5] A.J. Dragt, Phys. Rev. Lett. 75, p. 1946 (1995). 